\title{
WWOX suppresses autophagy for inducing apoptosis in methotrexate-treated human squamous cell carcinoma
}

\author{
C-W Tsai ${ }^{1,14}$, F-J Lai ${ }^{2,14}$, H-M Sheu ${ }^{3}$, Y-S Lin ${ }^{1,4}$, T-H Chang ${ }^{5}$, M-S Jan ${ }^{6}$, S-M Chen ${ }^{7,8}$, P-C Hsu', T-T Huang ${ }^{1}$, T-C Huang ${ }^{1}$, M-C Sheen ${ }^{9}$, \\ S-T Chen ${ }^{10}$, W-C Chang ${ }^{4,11}$, N-S Chang ${ }^{4,12,13}$ and L-J Hsu ${ }^{*, 1,4,7,8}$
}

Squamous cell carcinoma (SCC) cells refractory to initial chemotherapy frequently develop disease relapse and distant metastasis. We show here that tumor suppressor WW domain-containing oxidoreductase (WWOX) (also named FOR or WOX1) regulates the susceptibility of SCC to methotrexate (MTX) in vitro and cure of SCC in MTX therapy. MTX increased WWOX expression, accompanied by caspase activation and apoptosis, in MTX-sensitive SCC cell lines and tumor biopsies. Suppression by a dominant-negative or small interfering RNA targeting WWOX blocked MTX-mediated cell death in sensitive SCC-15 cells that highly expressed WWOX. In stark contrast, SCC-9 cells expressed minimum amount of WWOX protein and resisted MTX-induced apoptosis. Transiently overexpressed WWOX sensitized SCC-9 cells to apoptosis by MTX. MTX significantly downregulated autophagy-related Beclin-1, Atg12-Atg5 and LC3-II protein expression and autophagosome formation in the sensitive SCC-15, whereas autophagy remained robust in the resistant SCC-9. Mechanistically, WWOX physically interacted with mammalian target of rapamycin (mTOR), which potentiated MTX-increased phosphorylation of mTOR and its downstream substrate p70 S6 kinase, along with dramatic downregulation of the aforementioned proteins in autophagy, in SCC-15. When WWOX was knocked down in SCC-15, MTX-induced mTOR signaling and autophagy inhibition were blocked. Thus, WWOX renders SCC cells susceptible to MTX-induced apoptosis by dampening autophagy, and the failure in inducing WWOX expression leads to chemotherapeutic drug resistance.

Cell Death and Disease (2013) 4, e792; doi:10.1038/cddis.2013.308; published online 5 September 2013

Subject Category: Cancer

Chemoresistance is a major obstacle to successful treatment of human head and neck squamous cell carcinoma (SCC). A significant proportion of patients with relapsed SCC after chemotherapy ultimately succumb to metastatic disease. ${ }^{1}$ The majority of anticancer drugs target rapidly proliferating cells and induce cell death by means of apoptosis. Methotrexate (MTX) is a folate antagonist that inhibits dihydrofolate reductase (DHFR) and thymidylate synthase (TS) in the folate cycle and the pathway of de novo biosynthesis of purine nucleotides from ribose 5-phosphate. ${ }^{2}$ MTX is commonly used in cancer chemotherapy. ${ }^{1}$ Although MTX is relatively effective in the initial treatment for patients with SCC, the selection of cancer cells that are refractory to the cytotoxic effect of MTX may lead to the development of advanced SCC or unfavorable relapse. ${ }^{1}$ Despite extensive efforts, the molecular mechanisms underlying MTX resistance in SCC cells are not fully elucidated. An urgent need exists for the development of a new strategy for targeted cancer therapy.

Autophagy is a conserved intracellular catabolic process that degrades cytoplasmic components through a lysosomal pathway. ${ }^{3}$ Autophagy is induced under stress conditions, such as nutrient starvation, hypoxia, heat and drug treatment.

\footnotetext{
${ }^{1}$ Department of Microbiology and Immunology, National Cheng Kung University Medical College, Tainan, Taiwan; ${ }^{2}$ Department of Dermatology, Chi-Mei Medical Center, Tainan, Taiwan; ${ }^{3}$ Department of Dermatology, National Cheng Kung University Medical College, Tainan, Taiwan; ${ }^{4}$ Center of Infectious Disease and Signaling Research, National Cheng Kung University, Tainan, Taiwan; ${ }^{5}$ Institute of Basic Medical Sciences, National Cheng Kung University Medical College, Tainan, Taiwan; ${ }^{6}$ Institute of Microbiology and Immunology, Chung Shan Medical University, Taichung, Taiwan; ${ }^{7}$ Department of Medical Laboratory Science and Biotechnology, National Cheng Kung University Medical College, Tainan, Taiwan; ${ }^{8}$ Research Center for Medical Laboratory Biotechnology, National Cheng Kung University Medical College, Tainan, Taiwan; ${ }^{9}$ Department of Surgery, Kaohsiung Medical University Hospital, Kaohsiung, Taiwan; ${ }^{10}$ Department of Cell Biology and Anatomy, National Cheng Kung University Medical College, Tainan, Taiwan; ${ }^{11}$ Graduate Institute of Medical Sciences, Taipei Medical University, Taipei, Taiwan; ${ }^{12}$ Institute of Molecular Medicine, National Cheng Kung University Medical College, Tainan, Taiwan and ${ }^{13}$ Department of Neuroscience and Physiology, SUNY Upstate Medical University, Syracuse, NY, USA

*Corresponding author: L-J Hsu, Department of Medical Laboratory Science and Biotechnology, National Cheng Kung University Medical College, 1 University Road, Tainan 70101, Taiwan. Tel: +886 6 2353535, ext 5765; Fax: +886 6236 3956; E-mail: hsu.lijin@ gmail.com

${ }^{14}$ These authors contributed equally to this work.

Keywords: tumor suppressor; methotrexate; chemotherapy; autophagy; apoptosis

Abbreviations: 5-FU, 5-fluorouracil; DHFR, dihydrofolate reductase; dnWWOX, dominant-negative mutant WWOX; E64d, (2S,3S)-trans-epoxysuccinyl-L-leucylamido3-methylbutane ethyl ester; GFP, green fluorescent protein; IRES, internal ribosome entry site; LC3, microtubule-associated protein light chain 3; LOH, loss of heterozygosity; mTOR, mammalian target of rapamycin; mTORC1, mTOR complex 1; MTX, methotrexate; p70S6K, p70 S6 kinase; PE, phosphatidylethanolamine; SCC, squamous cell carcinoma; shRNA, small hairpin RNA; siRNA, small interfering RNA; TS, thymidylate synthase; TUNEL, terminal deoxynucleotidyl transferase dUTP nick-end labeling; Tyr33, tyrosine 33; WWOX, WW domain-containing oxidoreductase

Received 24.2.13; revised 18.6.13; accepted 12.7.13; Edited by GM Fimia
} 
In some situations, autophagy has been implicated in type II (non-apoptotic) programmed cell death. ${ }^{4}$ Using growth factordependent cells from $\mathrm{Bax}^{-1-} \mathrm{Bak}^{-1-}$ mice, previous study has demonstrated that autophagy is essential for maintaining cell survival following growth factor withdrawal. ${ }^{5}$ During starvation, cells maintain ATP production and generate necessary amino acids from catabolism of intracellular constituents through autophagy. ${ }^{3}$ Autophagy may facilitate the survival of rapidly dividing cancer cells that have outgrown their vascular supply and encounter hypoxia or metabolic stress. ${ }^{6}$ Recent studies have suggested that autophagy has important roles in chemoresistance of cancer cells to some antimetabolic agents. ${ }^{7,8}$ Accumulating evidence has demonstrated that inhibition of autophagy increases the susceptibility of cancer cells to cytotoxic chemotherapy. ${ }^{9,10}$ Increased phosphorylation of mammalian target of rapamycin (mTOR) has been shown to be associated with diminished autophagy and increased resistance of pancreatic cancer cells to chemotherapeutic agents. ${ }^{11}$ Whether targeting autophagy can be exploited in cancer treatment remains controversial. The definitive evidence for the molecular mechanism by which autophagy helps cancer cells to fend off chemotherapeutic drugs is still lacking.

Human WWOX gene resides in a common fragile site FRA16D on chromosome 16q23.3-24.1. ${ }^{12}$ Frequent deletions, loss of heterozygosity $(\mathrm{LOH})$ and translocations of human WWOX gene have been found in numerous types of cancers. ${ }^{12}$ Poor prognosis or unfavorable clinical outcome in patients is associated with low or absent expression of WW domain-containing oxidoreductase (WWOX) protein in cancer specimens. ${ }^{13,14}$ Previous studies have shown that ectopically overexpressed WWOX inhibits the growth of lung, breast and pancreatic cancer cells in nude mice. ${ }^{15-17}$ Functional suppression of WWOX by dominant-negatives and small interfering RNA (siRNA) protects cells from apoptosis by tumor necrosis factor, staurosporine, ultraviolet light and ectopic p53 in vitro. ${ }^{12,18,19} \mathrm{~A}$ recent study showed higher incidence of spontaneous tumor formation in Wwox gene knockout mice, suggesting that WWOX/WOX1 is a bona fide tumor suppressor. ${ }^{20}$ We have previously demonstrated significant reduction of WWOX and its family proteins in poorly differentiated and metastatic cutaneous SCC without downregulation of WWOX mRNA, indicating a translational blockade of WWOX mRNA to protein. ${ }^{19}$ However, whether WWOX can be regarded as a prognostic marker for cancer chemotherapy remains uncertain.

Verrucous carcinoma is a distinctive variant of SCC. Previous studies showed that continuous intra-arterial infusion of MTX leads to complete cure of the disease. ${ }^{21-23}$ In this study, we determined that MTX infusion in SCC patients induced significant upregulation of WWOX protein expression along with caspase-3 activation and apoptosis in tumor biopsies. WWOX suppressed autophagy and enhanced MTX-induced apoptosis in SCC cells. The failure to induce WWOX protein upregulation was associated with MTX resistance in SCC cells. Clearly, these results suggest an effective role of WWOX in cancer chemotherapy and its potential as a biomarker for clinical prognosis.

\section{Results}

MTX induces apoptotic cell death in SCC-4 and SCC-15, but not SCC-9 cells. Three SCC cell lines derived from tongue biopsies ${ }^{24}$ were tested for their susceptibility to MTX. Our results showed that MTX-induced cell death in SCC-4 and SCC-15 cells in both time- and dose-dependent manners (Figures 1a and b). In stark contrast, SCC-9 cells were resistant to MTX treatment with doses as high as $800 \mu \mathrm{g} / \mathrm{ml}$ (Figure 1a). We assessed whether MTX induces apoptosis in SCC-15 cells by detecting the binding of annexin $\mathrm{V}$ to cell surface phosphatidylserine. Our data showed that MTX treatment significantly induced surface phosphatidylserine exposure in SCC-15 cells (Figure 1c). No increases in annexin V staining were observed in SCC-9 cells following MTX treatment (Figure 1c). Similarly, condensed nuclei and DNA fragmentation were increased in MTXtreated SCC-15, but not SCC-9 cells, as determined by 4'-6-diamidino-2-phenylindole staining and DNA ladders in agarose gel electrophoresis, respectively (Supplementary Figures 1 and 2).

Chemotherapeutic drug-induced cell death is associated with WWOX expression in SCC cells. Previous studies have shown that tumor suppressor WWOX sensitizes cells to apoptosis under stress conditions. ${ }^{18,25}$ To investigate the role of WWOX in chemotherapy, we examined the expression levels of WWOX in these SCC cells. Interestingly, the extent of cell death among three SCC cell lines positively correlated with their endogenous protein levels of WWOX (Figure 1d). Among the cell lines tested, SCC-15 cells expressed the highest amount of WWOX protein and exhibited the greatest susceptibility to MTX (Figures 1a and d). In contrast, MTX-resistant SCC-9 cells expressed minimum protein level of WWOX (Figure 1d). MTX upregulated the expression of WWOX protein in SCC-4 and SCC-15 cells in a dose-dependent manner (Figure 1e). Also, WWOX mRNA levels were upregulated upon MTX treatment in SCC-15 cells, as determined by reverse transcription PCR (Figure 1f) and real-time PCR analysis (Figure 1g). However, MTX did not increase the levels of WWOX protein and mRNA in SCC-9 cells (Figures 1e and f). MTX-induced upregulation of WWOX protein expression was accompanied by the sequential activation of caspase- 9 and caspase-3 in SCC-15 cells, as evidenced by reduction of pro-caspases and increase of their cleavage forms (Figure 1h).

Previous studies showed that continuous intra-arterial infusion of MTX resulted in complete tumor regression in patients with verrucous carcinoma, a distinctive variant of SCC. ${ }^{21-23}$ Notably, MTX treatment upregulated WWOX protein expression along with caspase-3 activation and apoptotic cell death (terminal deoxynucleotidyl transferase dUTP nick-end labeling (TUNEL)-positive staining) in the SCC tumor biopsies (Figures $1 \mathrm{i}$ and $\mathrm{j}$ ). To further confirm the role of WWOX in anticancer therapy, a widely used chemotherapeutic antimetabolite 5-fluorouracil (5-FU) was also tested. Although SCC- 9 cells were refractory to MTX treatment, these cells were susceptible to 5-FU-mediated cell death by upregulation of WWOX protein in cells (Supplementary Figure 3). 
a

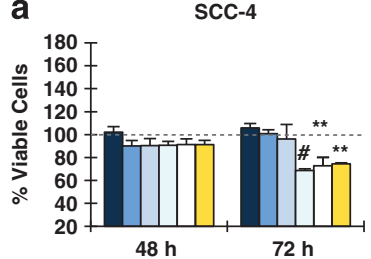

SCC-9

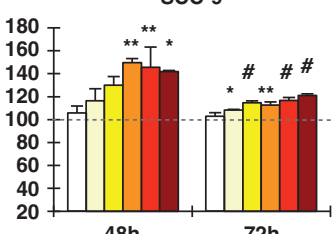

$72 \mathrm{~h}$

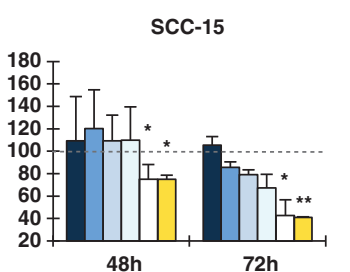

$72 \mathrm{~h}$

b $\operatorname{MTX}(10 \mu \mathrm{g} / \mathrm{ml})$

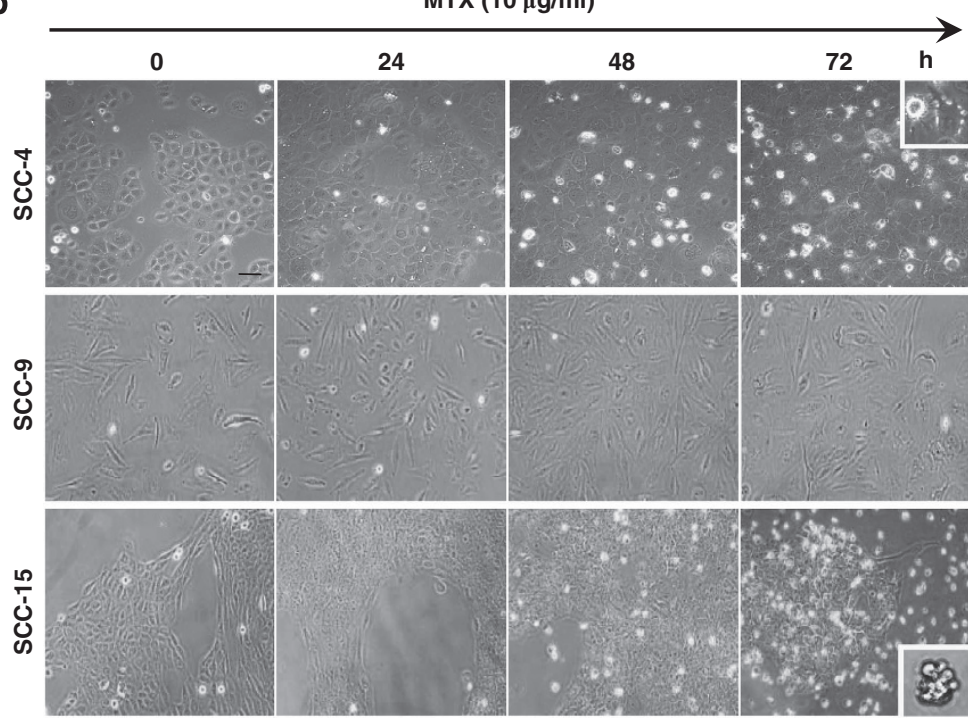

C

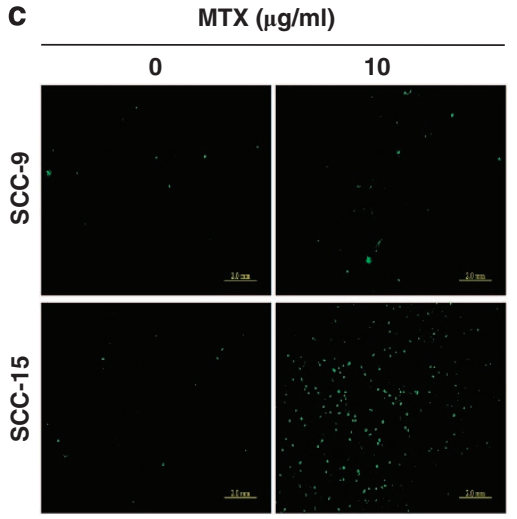

d

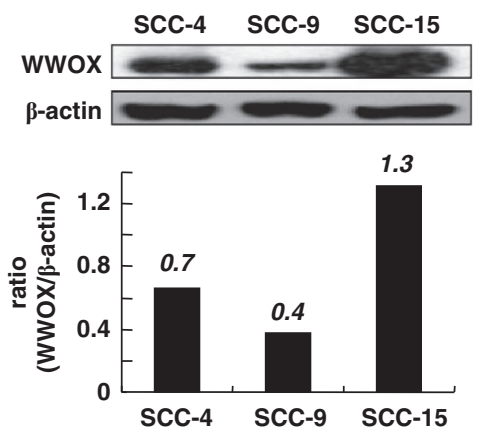

Figure 1 MTX-induced apoptotic cell death positively correlates with WWOX expression in SCC cells. (a) SCC-4, SCC-9 and SCC-15 cells were seeded on 96-well cell culture plates. One day later, cells were treated with the indicated doses of MTX and cultured for 48 and $72 \mathrm{~h}$, respectively. Cell viability was determined by crystal violet staining. The percentages of viable cells after MTX treatment were measured versus untreated control cells as described in the Methods. Representative results of six independent experiments are shown. (b) SCC-4, SCC-9 and SCC-15 cells were treated with $10 \mu \mathrm{g} / \mathrm{ml}$ MTX for the indicated times, followed by examining cell morphology under a light microscope $(\times 200)$. Enlarged are representative dead cells (see the insert). Dead cells are visualized mainly as 'whitish punctates' in the pictures. Scale bar, $60 \mu \mathrm{m}$. (c) MTX-induced phosphatidylserine exposure on the surface of SCC-15, but not SCC-9 cells. SCC-9 and SCC-15 cells were treated with or without MTX (10 $\mu \mathrm{g} / \mathrm{ml})$ for $72 \mathrm{~h}$. The binding of annexin V-FITC to phosphatidylserine on the cell surface was examined using a fluorescence microscope. (d) Western blot analysis of lysates from SCC-4, SCC- 9 and SCC-15 cells was performed to determine the expression levels of endogenous WWOX protein. The specific bands in the western blots were quantified using a densitometer. The bar graph depicts the ratio of WWOX to $\beta$-actin protein levels in SCC-4, SCC-9 or SCC-15 cells, respectively. (e) SCC-4, SCC-9 and SCC-15 cells were treated with the indicated doses of MTX for $72 \mathrm{~h}$. WWOX protein levels were determined using western blotting. $\beta$-Actin was used as an internal control. (f) SCC-15 and SCC-9 cells were treated with $10 \mu \mathrm{g} / \mathrm{ml}$ MTX and cultured for various time intervals. Total RNA was extracted from the SCC cells using TRIZOL reagent. The MRNA levels of WWOX and $\beta$-actin were determined by reverse transcription PCR. The PCR products were visualized on $1.2 \%$ agarose gels stained by ethidium bromide and quantified using a densitometer. The bar graphs depict the ratio of WWOX to $\beta$-actin mRNA levels in SCC-15 (upper panel) and SCC-9 cells (lower panel). (g) SCC-15 and SCC-9 cells were treated with $10 \mu \mathrm{g} / \mathrm{ml} \mathrm{MTX} \mathrm{for} 48$ or $72 \mathrm{~h}$. Quantification of WWOX mRNA expression in MTX-treated cells was performed by real-time PCR analysis, and data were analyzed using the comparative Ctmethod. (h) SCC-15 cells were treated with $10 \mu \mathrm{g} / \mathrm{ml}$ MTX and cultured for 24,48 and $72 \mathrm{~h}$. WWOX protein expression and the cleavage of caspase-9 and -3 were determined using western blotting. $\beta$-Actin was used as a loading control. (i) MTX-induced upregulation of WWOX protein is associated with tumor regression in patients with SCC. Continuous intra-arterial infusion of MTX (50 mg/day, 4-11 days) caused complete tumor regression in SCC patients. ${ }^{21-23}$ Cancer cells in the SCC tumor sections showed condensed chromatin within the shrinking cancer areas after intra-arterial MTX infusion (arrows in the upper right panel; H\&E stain). WWOX protein expression was markedly upregulated in the cancer cells after MTX treatment, as determined by immunohistochemistry (lower panels). Scale bar, $100 \mu \mathrm{m}$. (j) SCC cancer cells with condensed chromatin in the tumor sections (arrows in i) showed active caspase-3 staining (upper panel) and positive TUNEL (lower panel) after intra-arterial MTX treatment. Scale bar, $20 \mu \mathrm{m}$. (a, f and $\mathbf{g}$ ) Data represent means \pm S.D. ( $n=4$ per group). Comparisons with untreated control were made by Student's t-test. ${ }^{*} P<0.05,{ }^{* *} P<0.01$ and ${ }^{\sharp} P<0.001$ 
e

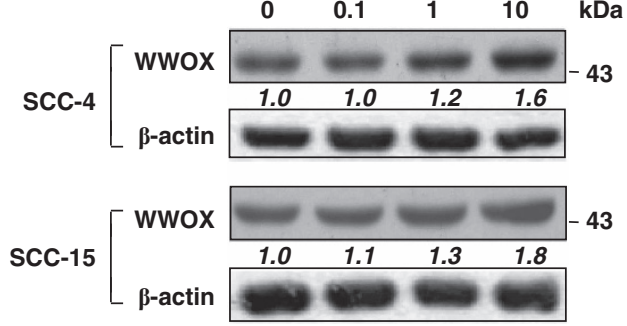

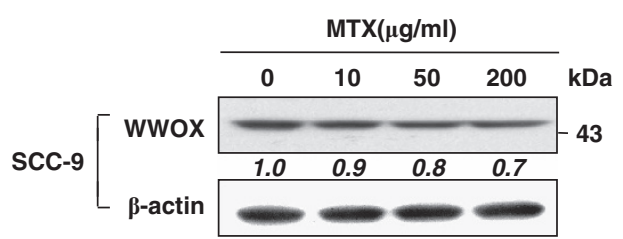

g

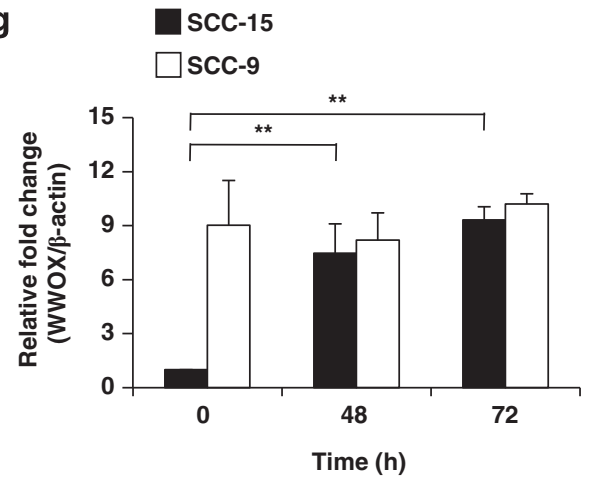

$\mathbf{f}$

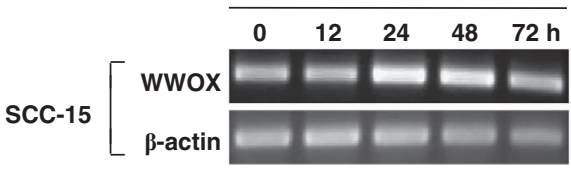

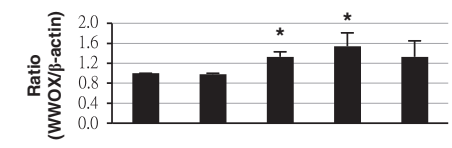

scc-9
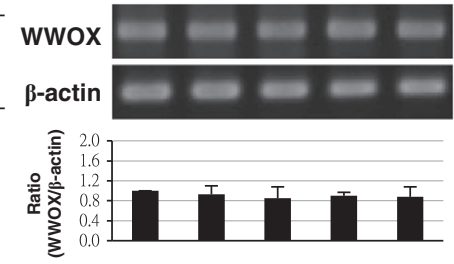

h
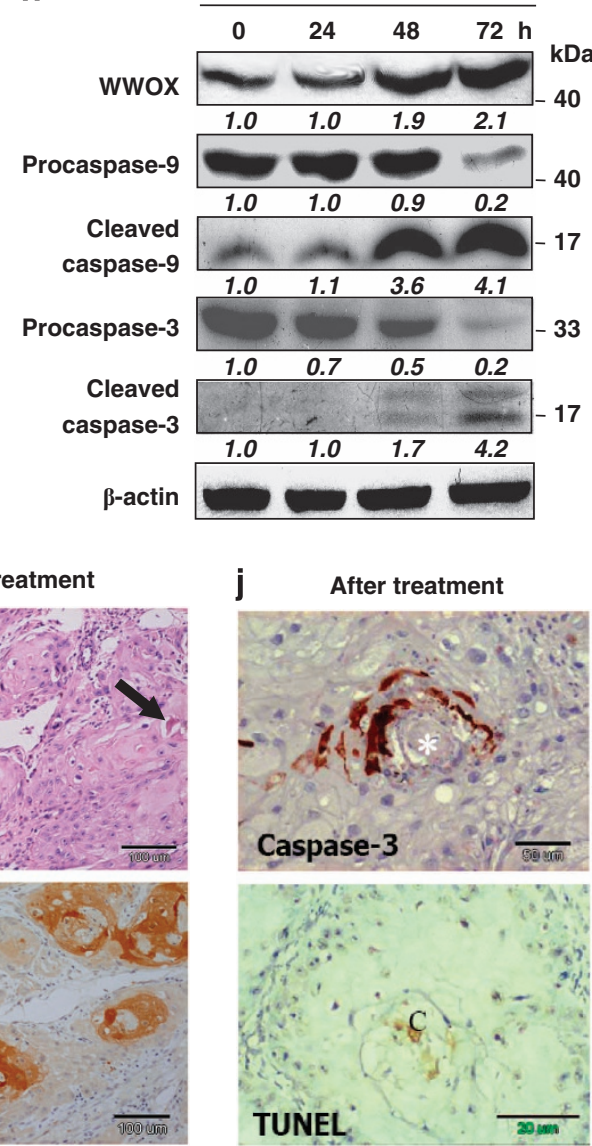

i

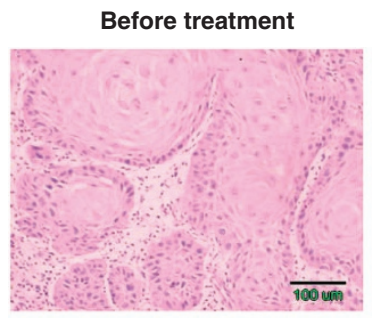

After treatment
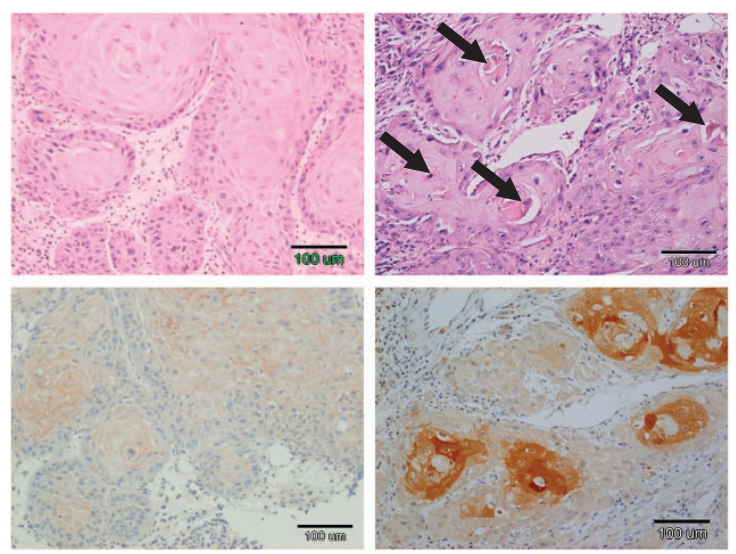

Figure 1 continued

Further, ectopic overexpression of WWOX protein in MTXresistant SCC-9 cells significantly enhanced MTX-induced apoptosis (Figures 2a and b). Conversely, siRNA knockdown of WWOX blocked MTX-mediated apoptosis in SCC-15 cells (Figures 2c and d). Tyrosine 33 (Tyr33) phosphorylation of WWOX protein has been suggested to be crucial for its functions. ${ }^{18}$ WWOX protein phosphorylation at Tyr33 was increased in SCC-15 cells after MTX treatment, but remained barely detectable in MTX-treated SCC-9 cells (data not shown). Transient expression of a dominant-negative mutant WWOX (dnWWOX) (containing a Tyr33 to Arg33 substitution) suppressed MTX-mediated cell death in SCC-15 cells (Figure 2e). Together, our results indicate that WWOX has a crucial role in the induction of SCC cell apoptosis by anticancer drugs, suggesting that failure in inducing WWOX expression results in chemoresistance in SCC. 


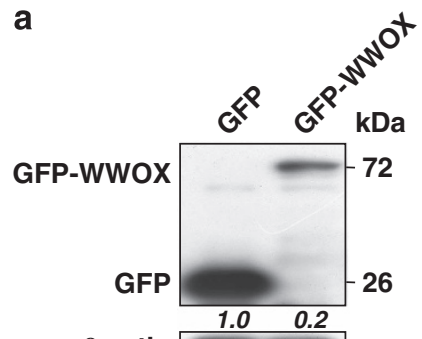

$\beta$-actin

b
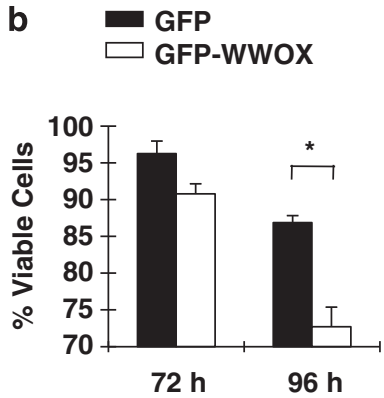

C

C

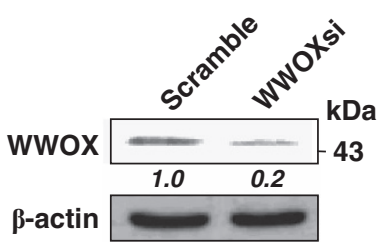

d

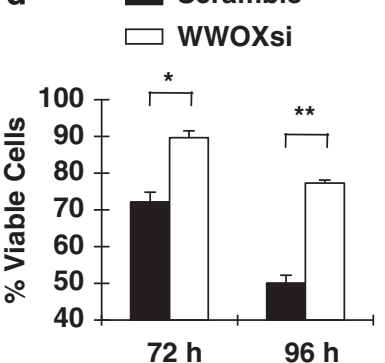

e

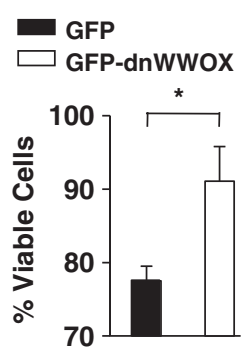

Figure 2 WWOX is crucial in MTX-induced SCC cell death. (a and b) Ectopic overexpression of WWOX in SCC-9 cells increased MTX-induced death. (a) SCC-9 cells were transfected with a vector encoding GFP-tagged human WWOX (GFP-WWOX) or a control GFP vector by electroporation. After incubating at $37^{\circ} \mathrm{C}$ for $24 \mathrm{~h}$, total lysates of SCC-9 cells were examined for expression of GFP or GFP-WWOX using western blotting using an anti-GFP antibody. All transfected proteins were detected at their expected molecular weight. $\beta$-Actin was used as a loading control. (b) Twenty-four hours post transfection, SCC-9 cells expressing GFP or GFP-WWOX were treated with or without $10 \mu \mathrm{g} / \mathrm{ml} \mathrm{MTX}$ and cultured for an additional 72 or $96 \mathrm{~h}$. Cell viability was determined following MTX treatment by crystal violet staining. The percentages of viable cells after MTX treatment were measured versus untreated control cells as described in the Materials and Methods. (c-e) MTX-induced apoptosis in SCC-15 cells was suppressed by either WWOXsi or dnWWOX mutant. (c) SCC-15 cells were transfected with an siRNA construct targeting WWOX (WWOXsi; in pSuppressorNeo) or a control scrambled sequence by electroporation. After culturing for $48 \mathrm{~h}$, WWOX protein levels in the SCC-15 cell lysates were examined using western blotting. $\beta$-Actin was used as a loading control. (d) Twenty-four hours after transfection, the WWOXsi or scrambled siRNA-transfected SCC-15 cells were treated with or without $10 \mu \mathrm{g} / \mathrm{ml}$ MTX and cultured for an additional 72 or $96 \mathrm{~h}$. The percentages of viable cells after MTX treatment were measured by crystal violet staining. (e) SCC-15 cells were transfected with a vector encoding GFP-tagged dnWWOX (GFP-dnWWOX) or a control GFP vector by electroporation. After incubating for $24 \mathrm{~h}$, cells were treated with or without $10 \mu \mathrm{g} / \mathrm{ml}$ MTX and cultured for an additional $96 \mathrm{~h}$. The percentages of viable cells after MTX treatment were measured by crystal violet staining. $(\mathbf{b}, \mathbf{d}$ and $\mathbf{e})$ Data are shown as means \pm S.D. ( $n=4$ per group). Representative results of three to five independent experiments are shown. Comparisons were made by Student's $t$-test. ${ }^{*} P<0.05$ and ${ }^{* \star} P<0.01$ compared with untreated control

MTX modulates autophagy in SCC cells. Autophagy constitutes one of the major responses to cellular stress. ${ }^{6}$ Although the role of autophagy in life-and-death decisions of cancer cells is controversial, an emerging body of evidence supports that autophagy promotes cancer cell survival under conditions of hypoxia, metabolic stress and chemoresistance. ${ }^{6,10,26}$ To investigate whether autophagy is associated with MTX susceptibility of SCC cells, we examined the expression of autophagy essential proteins in both MTX-sensitive SCC-15 and -resistant SCC-9 cells. Our results showed that MTX increased Beclin-1 protein expression in SCC- 9 cells as early as $12 \mathrm{~h}$, followed by reduction to the basal level in $24 \mathrm{~h}$ (Figure $3 \mathrm{a}$ ). A slight increase in the abundance of Atg12-Atg5 conjugate was observed following MTX treatment in SCC-9 cells (Figure 3a). In contrast, the expression levels of Beclin-1 and Atg12-Atg5 were evidently downregulated by MTX treatment in SCC-15 cells (Figure 3a). Interestingly, we found that the level of $21-\mathrm{kDa}$ Atg12 was significantly downregulated following MTX treatment in SCC-15 cells (Supplementary Figure 4). This may lead to the reduction of Atg12-Atg5 conjugate $(55 \sim 60 \mathrm{kDa})$ and the increased amount of $32 \mathrm{kDa}$-free Atg5 in MTX-treated SCC-15 cells (Figure $3 a$ and Supplementary Figure 4). In addition, using an anti-Atg12 antibody, we found that the reduction of Atg12-Atg5 conjugate was accompanied by an increase in the amount of $\sim 50 \mathrm{kDa}$ protein (see asterisk in Supplementary Figure 4). Whether this protein is a cleaved form of Atg12-Atg5 in MTX-treated SCC-15 cells needs further verification. Clearly, these observations indicate that MTX treatment upregulates the expression of essential proteins for autophagy in MTX-resistant SCC-9 cells, whereas suppresses autophagic protein expression in MTX-sensitive SCC-15 cells.

A morphological hallmark of autophagy is the presence of autophagosomes that have a double-membrane structure and contain cytoplasmic cargo to be degraded., ${ }^{3,27}$ Microtubule-associated protein light chain 3 (LC3) protein is a posttranslational modifier required for autophagosome formation. During autophagy, cytoplasmic LC3 protein (LC3-I) is conjugated to phosphatidylethanolamine (PE) by an ubiquitinlike system. After PE conjugation, LC3 redistributes from a diffuse pattern in the cytoplasm to a punctate structure on the autophagosome membranes. ${ }^{27}$ Thus, lipidated LC3 (LC3-II) is now widely used as a marker to monitor autophagy. To test whether MTX treatment regulates autophagy in SCC cells, we determined the changes in LC3 amounts using western blotting. Our results clearly demonstrated that endogenous 
a
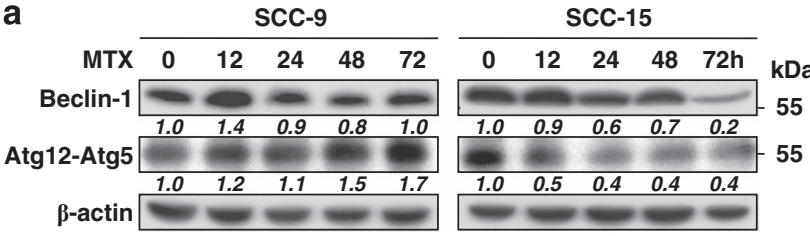

b
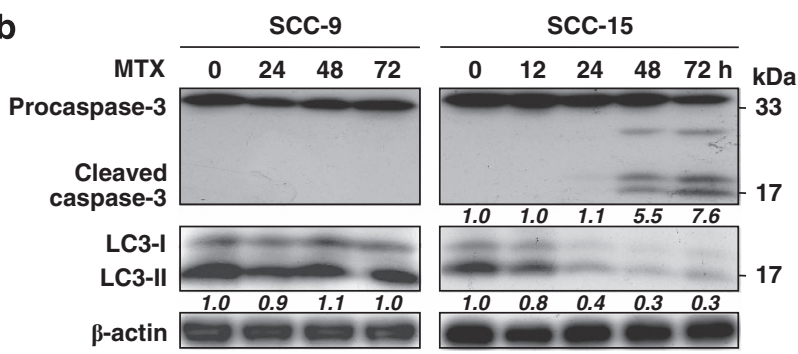

C

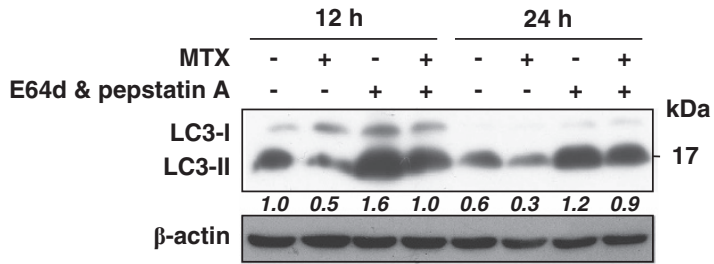

Figure 3 MTX downregulates the expression of Beclin-1, Atg12-Atg5 and LC3 in SCC-15 cells, but increases their levels in SCC-9 cells. (a) Total protein extracts were prepared from SCC-9 and SCC-15 cells after treatment with $10 \mu \mathrm{g} / \mathrm{ml} \mathrm{MTX} \mathrm{for}$ the indicated time intervals. The expression levels of Beclin-1 and Atg12-Atg5 conjugate were determined using western blotting. $\beta$-Actin was used as an internal control. (b) SCC-9 and SCC-15 cells were treated with $10 \mu \mathrm{g} / \mathrm{ml}$ MTX. Cytosolic protein extracts were prepared at various time points. LC3 protein expression and caspase- 3 cleavage were assessed using western blotting. $\beta$-Actin was used as an internal control. (c) SCC-15 cells were treated with or without $10 \mu \mathrm{g} / \mathrm{ml}$ MTX in the presence or absence of E64d $(30 \mu \mathrm{m})$ and pepstatin $\mathrm{A}(15 \mu \mathrm{m})$. After culturing for 12 or $24 \mathrm{~h}$, cytosolic protein extracts were analyzed for LC3 protein expression using western blotting. $\beta$-Actin was used as an internal control

LC3 expression was significantly downregulated by MTX treatment in SCC-15 cells along with caspase-3 activation, but remained unchanged in MTX-treated SCC-9 cells (Figure 3b). We did not detect cleaved forms of caspase-3 in SCC-9 cells following MTX treatment (Figure 3b). After autophagosome formation, the fusion of autophagosome with lysosome leads to the degradation of materials in autophagic vacuoles including LC3. To exclude the possibility that the decrease in LC3 protein expression in MTXtreated SCC-15 cells could be due to increased autophagic flux, ${ }^{27}$ we used protease inhibitors E64d and pepstatin A to inhibit lysosomal enzymes in the autophagic vacuoles. Our data revealed that MTX treatment decreased LC3-II protein expression in SCC-15 cells at $12 \mathrm{~h}$, and the presence of E64d and pepstatin A did not prevent MTX-induced downregulation of LC3 protein expression (Figure 3c), suggesting that the reduction of LC3 protein levels in MTX-treated SCC-15 cells is not caused by rapid lysosomal turnover. Treatment of SCC-15 cells with a proteasome inhibitor MG132 blocked MTX-induced LC3 protein downregulation, indicating that LC3 protein is degraded via the ubiquitin/ proteasomal pathway post MTX treatment (Supplementary Figure 5).
To detect autophagosomes, accumulation of green fluorescent protein (GFP)-tagged LC3-II (GFP-LC3) at the autophagosomal membranes was monitored by fluorescence microscopy. Typical cytoplasmic GFP-LC3 puncta were observed in the SCC-15 cells transiently transfected with GFP-fused LC3 CDNA, indicating active autophagy in SCC-15 cells (Figure 4A). The amounts of GFP-LC3 puncta were markedly reduced in MTX-treated SCC-15 cells, as compared with the untreated SCC-15 cells (Figure 4A). In contrast, the percentage of cells with punctate GFP-LC3 fluorescence and the average numbers of GFP-LC3 punctate structures per cell remained unchanged in the SCC- 9 cells after MTX treatment (Figure 4A). To further verify the above observations, the appearance of cytoplasmic vacuoles related to autophagosomes was examined by transmission electron microscopy. In agreement with our findings using fluorescence microscopy, numerous autophagosomes with double-membrane structure were examined in SCC-15 cells before MTX treatment (Figure 4B). Our data clearly demonstrated that the cytoplasmic autophagosomes disappeared upon treatment of SCC-15 cells with MTX (Figure 4B). Instead, a large number of mitochondria with swollen cristae were found in the MTXstimulated SCC-15 cells. In contrast, as compared with the untreated SCC-9 cells, autophagosome formation remained unaffected in MTX-treated SCC-9 cells (Figure 4B). Together, we determined that, following MTX treatment, autophagy was suppressed in MTX-sensitive SCC-15 and SCC-4 cells, but not in MTX-resistant SCC-9 cells, suggesting that inhibition of autophagy may sensitize SCC cancer cells to chemotherapeutic drugs.

MTX treatment modulates mTOR signaling in SCC cells via WWOX. The mTOR complex 1 (mTORC1) is a cellular sensor for nutritional conditions that negatively regulates autophagy. ${ }^{28}$ Inhibition of mTORC1 activity induces autophagy in many types of cells. To study whether MTX treatment regulates mTORC1 activity in SCC cells, we examined protein phosphorylation of $\mathrm{mTOR}$ and its downstream effector p70 S6 kinase (p70S6K) at Ser2448 and Thr389, respectively. Our data showed that MTX stimulated protein phosphorylation of mTOR and p70S6K in SCC-15 cells after $24 \mathrm{~h}$ of treatment, whereas mTOR phosphorylation was downregulated in SCC-9 cells following MTX treatment (Figure 5a). Protein phosphorylation of p70S6K at Thr389 was barely detectable in SCC-9 cells (Figure 5a). Interestingly, we detected colocalization of WWOX with mTOR in SCC-15 cells by confocal microscopy (Supplementary Figure 6a). Co-immunoprecipitation analysis further confirmed their interaction in SCC-15 cells (Figure $5 \mathrm{~b}$ and Supplementary Figure 6b). Ectopic overexpression of WWOX in SCC-15 cells significantly augmented MTXinduced phosphorylation of mTOR and p70S6K, as compared with the GFP-transfected control cells (Figure $5 \mathrm{c}$ ). Conversely, siRNA-mediated knockdown of WWOX suppressed MTX-stimulated activation of the mTOR/p70S6K signaling pathway in SCC-15 cells (Figure $5 d$ ). Together, we determined that MTX stimulated $\mathrm{mTOR} / \mathrm{p} 70 \mathrm{~S} 6 \mathrm{~K}$ signaling in SCC-15 cells via WWOX, raising the possibility that WWOX may regulate autophagy through $\mathrm{mTOR}$ activation in MTX-treated SCC-15 cells. 
A
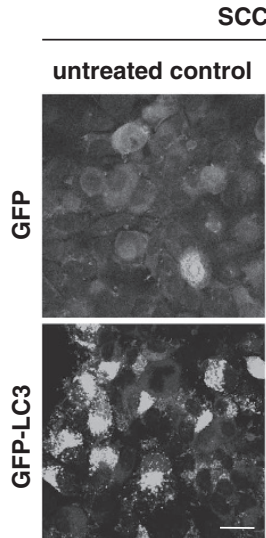

SCC-15
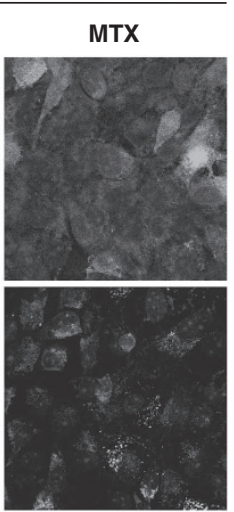

SCC-9

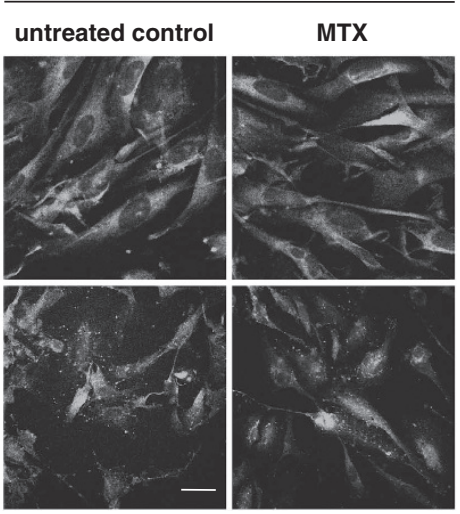

B

SCC-15
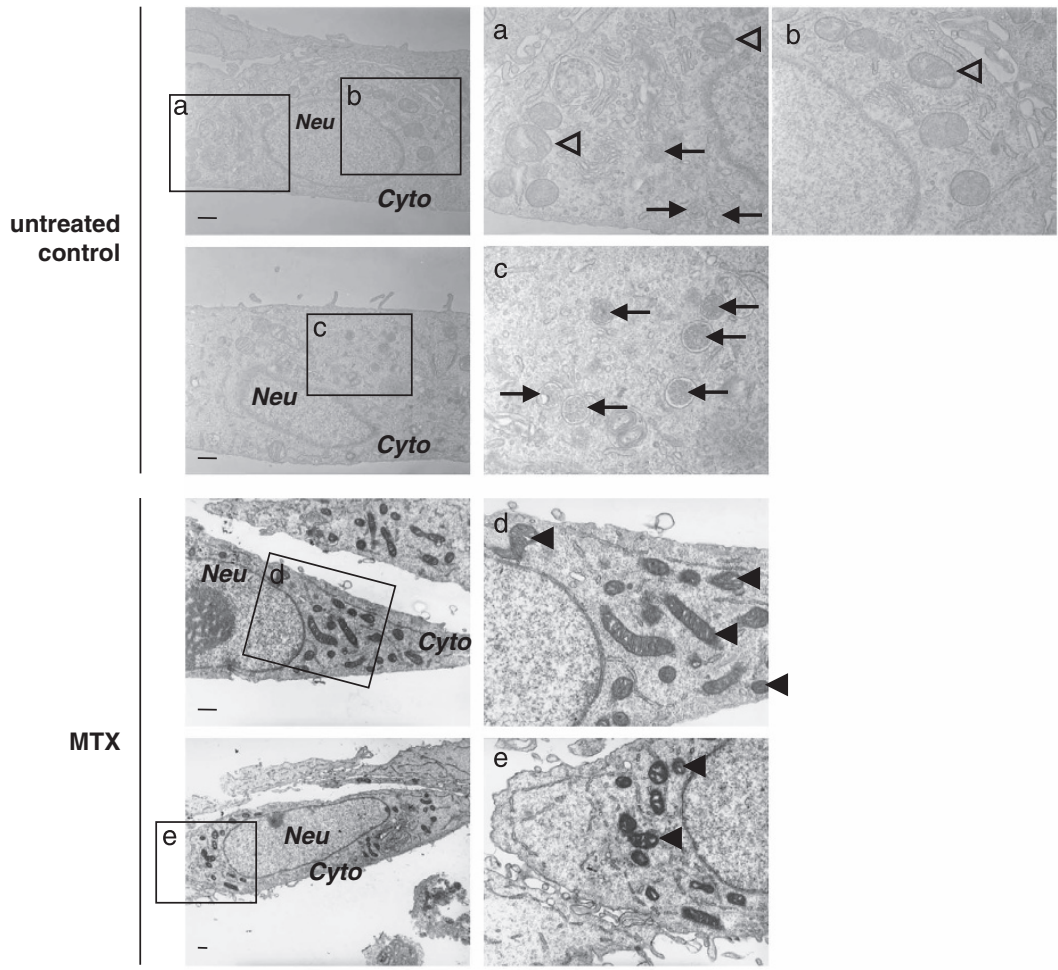

SCC-9
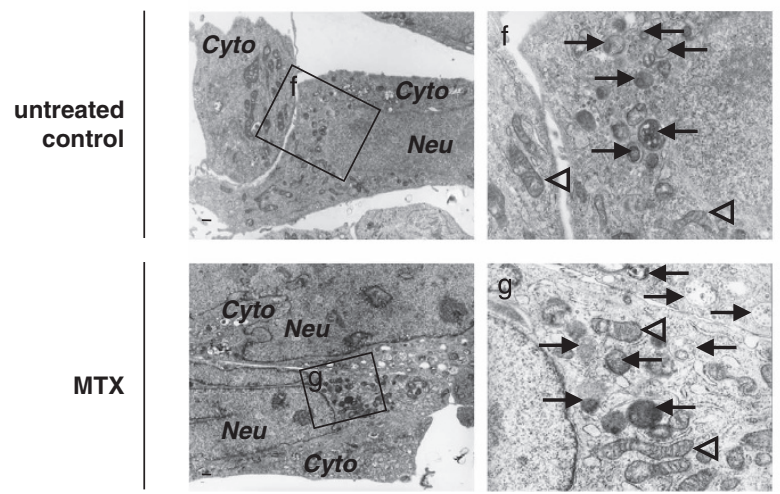
WWOX suppresses autophagy in SCC cells. To explore the role of WWOX in autophagy regulation, WWOX cDNA was transiently transfected into SCC-9 cells, and LC3-II protein expression in these cells following MTX treatment was examined using western blotting. Our results showed that ectopic overexpression of WWOX dramatically decreased the lipidated LC3-II protein expression in MTXtreated SCC-9 cells (Figure 6a). Lentiviral small hairpin RNA (shRNA)-mediated knockdown of WWOX expression partially prevented MTX-induced reduction of LC3-II in SCC-15 cells (Figure 6b). In our recently developed Wwox gene knockout mouse embryonic fibroblasts, increased expression of LC3-II protein was detected in the cytosolic protein extracts from the $W w o x^{+/-}$and $W w o x^{-1-}$ cells using western blotting, as compared with the $W_{w o x}{ }^{+/+}$control cells (Supplementary Figure 7). The ubiquitin-like autophagyrelated protein conjugation system has been demonstrated to be required for autophagosome formation. ${ }^{29}$ Previous studies showed that Atg12-Atg5 conjugate has an E3-like function to stimulate LC3 lipidation and has an essential role in autophagy induction. ${ }^{30}$ We detected significantly reduced amount of Atg12-Atg5 conjugate in the SCC-9 cells overexpressing WWOX, and MTX treatment further downregulated the expression level of Atg12-Atg5 conjugate in these cells (Figure 6c). Beclin-1 is a mammalian ortholog of the yeast Atg6 that has been shown to be required for the initiation of autophagy through its interaction with class III PI3-kinase/Vps34. ${ }^{31}$ Our results showed that ectopic overexpression of WWOX suppressed protein expression of Beclin-1 in both SCC-9 and SCC-15 cells (Figure 6d). Together, our results clearly demonstrate a role of WWOX in the regulation of autophagy in SCC cells.

\section{Discussion}

In summary, we have clearly demonstrated that WWOX is central to the MTX-induced apoptosis of SCC-15 cells, and this positively correlates with WWOX downregulation
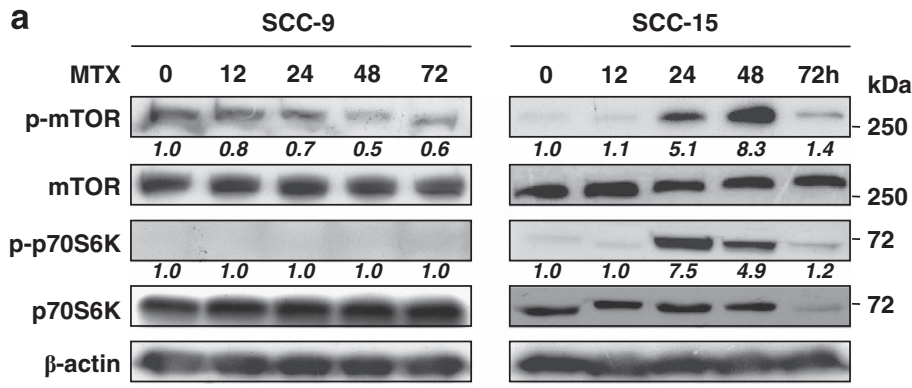

b
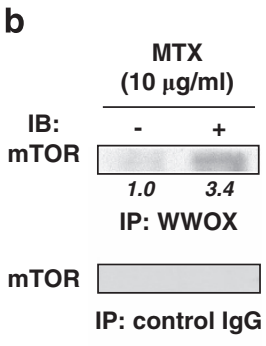

Input:

mTOR
C

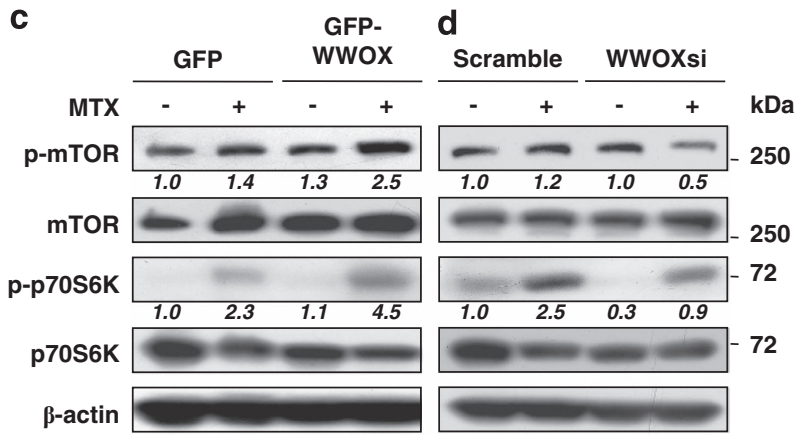

Figure 5 MTX upregulates the autophagy-inhibitor mTOR signaling in SCC-15 cells through WWOX. (a) MTX increased the phosphorylation of mTOR and its downstream p70S6K in SCC-15 cells, whereas suppressed mTOR phosphorylation in SCC-9 cells. SCC-9 and SCC-15 cells were treated with $10 \mu \mathrm{g} / \mathrm{ml}$ MTX for the indicated time intervals. Total protein extracts were prepared and analyzed for the phosphorylation of mTOR and p70S6K at Ser2448 and Thr389, respectively, using western blotting. $\beta$-Actin was used as a loading control. (b) By co-immunoprecipitation, WWOX was shown to interact with endogenous mTOR in SCC-15 cells. MTX increased their binding in the cells during treatment of $24 \mathrm{~h}$. (c) SCC-15 cells were transfected with a vector encoding GFP-WWOX or a control GFP vector by electroporation. After incubating at $37^{\circ} \mathrm{C}$ for $24 \mathrm{~h}$, cells were treated with or without $10 \mu \mathrm{g} / \mathrm{ml} \mathrm{MTX}$ and cultured for an additional $24 \mathrm{~h}$. Total lysates of SCC-15 cells were examined for phosphorylation of mTOR and p70S6K using western blotting. $\beta$-Actin was used as an internal control. (d) SCC-15 cells were transfected with WWOXsi or a control scrambled sequence by electroporation. Twenty-four hours after transfection, cells were treated with or without $10 \mu \mathrm{g} / \mathrm{ml}$ MTX. After culturing at $37^{\circ} \mathrm{C}$ for $24 \mathrm{~h}$, total cell lysates were examined for protein phosphorylation of mTOR and P70S6K using western blotting. $\beta$-Actin was used as a loading control

Figure 4 MTX suppresses autophagosome formation in SCC-15, but fails to inhibit autophagy in SCC-9 cells. (A) SCC-15 and SCC-9 cells were transiently transfected with GFP-tagged LC3-expressing construct or a control GFP vector by electroporation. After culturing for $18 \mathrm{~h}$, cells were treated with or without $10 \mu \mathrm{g} / \mathrm{ml}$ MTX. Twenty-four hours later, cells were fixed with $3.7 \%$ formalin/PBS at $4{ }^{\circ} \mathrm{C}$ for $15 \mathrm{~min}$ and examined for punctated GFP-LC3 protein by confocal fluorescence microscopy. Scale bar, $20 \mu \mathrm{m}$. (B) SCC-15 and SCC-9 cells were treated with or without $10 \mu \mathrm{g} / \mathrm{ml} \mathrm{MTX} \mathrm{for} 24 \mathrm{~h}$. The presence of autophagic vacuoles in these cells was monitored by transmission electron microscopy. The enlarged images at the right panel $(\mathrm{a}-\mathrm{g})$ are from the boxed areas in the left panel. Disappearance of autophagosomes (arrows) post MTX treatment was examined in SCC-15 cells. In comparison with the normal mitochondrial ultrastructure (open arrowheads) in untreated SCC-15 cells, numerous intensely stained mitochondria with swollen cristae (solid arrowheads) were observed in MTX-treated SCC-15 cells. In contrast, the autophagosome number and mitochondrial morphology remained largely unchanged after MTX treatment in SCC-9 cells. Original magnification: left panel images, $\times 10000$ or $\times 5000$. Scale bars, $1 \mu \mathrm{m}$. Cyto, cytoplasm; Neu, nucleus 
a

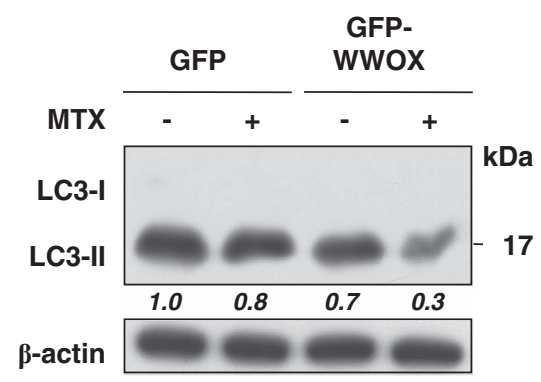

c

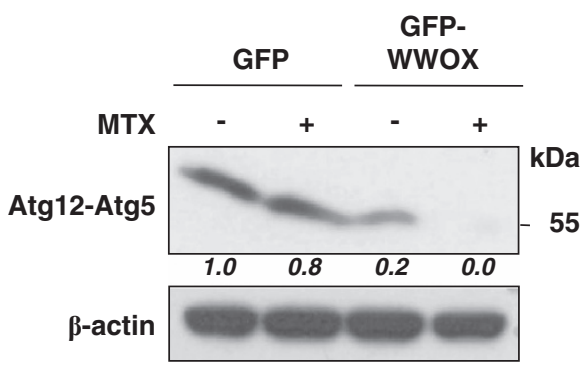

b

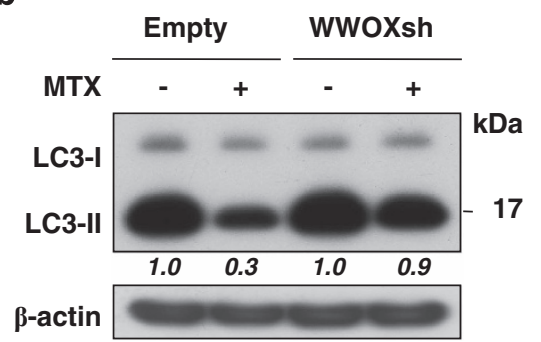

d

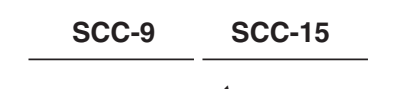

e

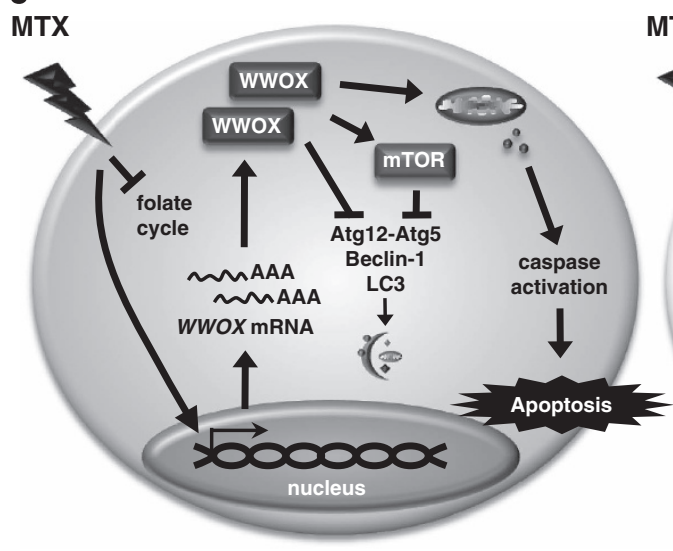

MTX-sensitive

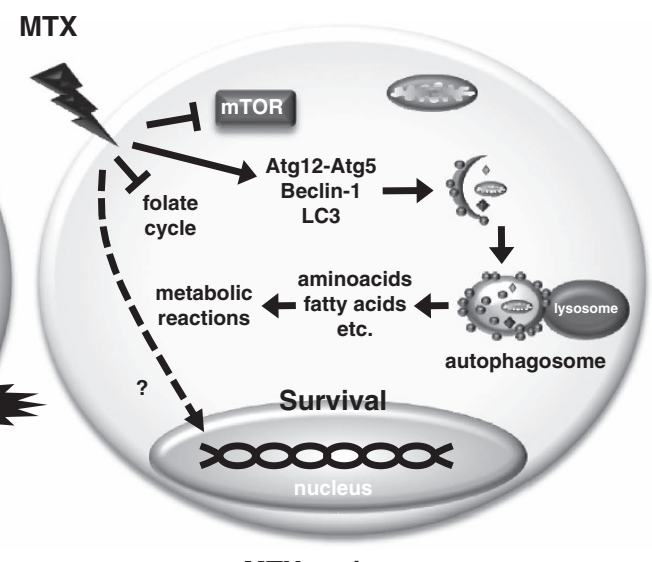

MTX-resistant

Figure 6 WWOX suppresses autophagy following MTX treatment. (a) SCC-9 cells were transfected with a vector encoding GFP-WWOX or a control GFP vector by electroporation. After incubating at $37^{\circ} \mathrm{C}$ for $24 \mathrm{~h}$, cells were treated with or without $10 \mu \mathrm{g} / \mathrm{ml} \mathrm{MTX}$ and cultured for an additional $24 \mathrm{~h}$. Total cell lysates were examined for LC3 protein expression using western blotting. $\beta$-Actin was used as a loading control. (b) SCC-15 cells were infected with empty lentivirus or lentivirus expressing WWOX shRNA. Two days after infection, SCC-15 cells were treated with or without $10 \mu \mathrm{g} / \mathrm{ml}$ MTX and cultured for an additional $24 \mathrm{~h}$. Total cell lysates were examined for LC3 protein expression using western blotting. $\beta$-Actin was used as a loading control. (c) SCC-9 cells were transfected with a vector encoding GFP-WWOX or a control GFP vector by electroporation. After incubating at $37^{\circ} \mathrm{C}$ for $24 \mathrm{~h}$, cells were treated with or without $10 \mu \mathrm{g} / \mathrm{ml} \mathrm{MTX}$ and cultured for an additional $48 \mathrm{~h}$. Total cell lysates were examined for the expression of Atg12-Atg5 protein conjugate using western blotting. $\beta$-Actin was used as a loading control. (d) SCC-9 and SCC-15 cells were transfected with a vector encoding GFP-WWOX or a control GFP vector by electroporation. After culturing for $24 \mathrm{~h}$, total cell lysates were analyzed for Beclin-1 protein expression using western blotting. $\beta$-Actin was used as a loading control. (e) Diagrams illustrating the potential effects of WWOX on MTX susceptibility. Left, elevated expression of WWOX renders SCC cells susceptible to MTX-induced apoptosis by suppressing autophagy. Right, SCC cells counteract MTX-induced metabolic stress by activating autophagy. Absent expression of WWOX is associated with resistance to chemotherapeutic drugs in cancer cells

of effector proteins in autophagy (Figure 6e). In contrast, the developed drug resistance is associated with upregulation of proteins in autophagy in SCC-9 cells (Figure 6e). Thus, WWOX suppresses autophagy for inducing apoptosis in MTX-treated human SCC. A clear strategy in cancer targeting is boosting WWOX for suppression of autophagy in treating cancers.

A recent study showed that WWOX inhibits $\Delta N p 63 \alpha$ function for sensitizing human osteosarcoma cell line Saos2 to cisplatin-induced apoptosis. ${ }^{32}$ The expression of WWOX has been suggested to be associated with a favorable outcome in breast cancer patients. ${ }^{33}$ Human WWOX gene spans the common chromosomal fragile site FRA16D. WWOX gene alterations, such as $\mathrm{LOH}$, deletions and translocations, have been frequently noted in numerous types of cancers. ${ }^{12}$ Previous studies suggested that epigenetic silencing of WWOX gene is associated with cancer development. ${ }^{34,35}$ Restoration of WWOX expression by either treatment of DNA methyltransferase and histone deacetylase inhibitors or transfection with adenovirus carrying WWOX cDNA suppresses cancer cell growth in vitro and in vivo. ${ }^{16,36}$ We have determined significant reduction or absent expression of WWOX protein in cutaneous SCC with moderate and poor differentiation. ${ }^{19}$ Interestingly, we determined that there 
is no significant difference in the WWOX mRNA levels between cutaneous SCC and the adjacent normal epidermis. ${ }^{19}$ Whether the reduction of WWOX protein levels in moderately and poorly differentiated SCC cells is due to a translational blockade of mRNA to protein or acceleration of protein degradation is unclear. Our results revealed that WWOX is crucial for MTX-induced SCC cell apoptosis, suggesting that downregulation of WWOX protein expression during SCC progression may lead to chemoresistance in cancer cells.

Here a barely detectable level of WWOX protein is shown in MTX-resistant SCC-9 cells. In stark contrast, quantitative realtime PCR data revealed that high level of WWOX mRNA is present in SCC-9 cells (Figure 1g). These results suggest that SCC- 9 cells may control the machinery of WWOX protein translation and/or degradation for preventing MTX-induced cell death. Indeed, we have identified an internal ribosome entry site (IRES) at the WWOX $5^{\prime}$ UTR and determined its activity by dicistronic reporter assay (unpublished data). Whether WWOX IRES-driven translation is impeded in MTX-resistant SCC-9 cells is currently under investigation. Furthermore, continuous intra-arterial infusion of MTX to patients with SCC induced WWOX protein upregulation and cancer cell apoptosis. ${ }^{21-23}$ In accordance with the clinical findings in SCC tumor biopsies, we determined increased mRNA and protein levels of WWOX and cell apoptosis in MTX-treated SCC-15 cells. In contrast, MTX treatment failed to increase WWOX mRNA and protein levels in SCC-9 cells, suggesting that aberrant signaling or epigenetic dysregulation of WWOX gene activation may also lead to the failure of WWOX protein upregulation in MTX-treated SCC- 9 cells. Reduced level of WWOX protein expression in breast cancers has been reported to be associated with tamoxifen resistance. ${ }^{37}$ The underlying mechanisms for the minimal expression of WWOX protein in drug-resistant cancer cells remain to be elucidated.

MTX is a potent inhibitor of DHFR and TS, two key enzymes in folate metabolism, thus preventing de novo purine biosynthesis. ${ }^{2}$ Defective MTX transport via reduced folate carrier and folate receptor or increased drug efflux via ATPbinding cassette subfamily transporters have been considered to be important mechanisms of cellular resistance to MTX. ${ }^{2}$ We determined that MTX-induced apoptosis in SCC-4 and SCC-15, but not SCC-9 cells, in both time- and dosedependent manners. The acquired resistance to apoptosis induced by MTX in SCC-9 cells might not be attributed to MTX transport alterations, because we detected a transient increase in SCC-9 cell growth after culturing for $48 \mathrm{~h}$ with increasing doses of MTX. It is unclear how intracellular MTX accumulation stimulates SCC-9 cell growth. Our data indicated that the basal autophagy is active in SCC-9 cells, as evidenced by the presence of numerous autophagosomes in the cytoplasm and a high level of LC3-II protein expression. Following MTX treatment, the protein expression levels of Beclin-1 and Atg12-Atg5 were upregulated, and autophagy remained robust in SCC-9 cells. These results suggest that SCC-9 cells may utilize the autophagic pathway as a survival mechanism to evade the perturbations of cellular biosynthetic processes by the antimetabolite MTX and sustain cell viability upon metabolic stress.
Control of autophagy by oncogenes and tumor-suppressor genes has been shown to be crucial for adaptation of cancer cells to stress. ${ }^{6}$ Modulating autophagy via manipulation of crucial gene expression may potentiate the antitumor effect of chemotherapeutic agents and provide a potential strategy for effective treatment. We show here that WWOX downregulated the expression levels of Atg12-Atg5 and Beclin-1 and suppressed autophagy in MTX-treated SCC cells. Interestingly, we determined that the reduction of LC3 protein via a proteasomal degradation pathway was associated with the presence of WWOX. Upon induction of autophagy, Beclin-1 interacts with class III PI3-kinase/Vps34, and this binding is central to the recruitment of autophagic proteins to the pre-autophagosomal structure for nucleation. ${ }^{31}$ The ubiquitin-like conjugation systems Atg12-Atg5 and LC3-II further facilitate elongation of the autophagosomal membranes. ${ }^{30}$ WWOX may impede the early stages of autophagy by reducing the amounts of Beclin-1, Atg12-Atg5 and LC3 proteins in SCC cells, thus sensitizing cancer cells to MTXinduced apoptosis. The molecular mechanisms underlying downregulation of essential proteins for autophagy by WWOX in SCC cells are largely unclear.

Also, we demonstrated that WWOX interacted with mTOR and stimulated the activation of mTOR signaling in MTXtreated SCC-15 cells. mTORC1 interacts with the ULK complex (composed of Atg13, FIP200, Atg101 and ULK) and inhibits its kinase activity and membrane targeting. ${ }^{38}$ The mammalian ULK complex regulates the recruitment of downstream Atg proteins to the autophagosome formation site and is essential for initiating autophagy. ${ }^{38}$ It is possible that WWOX indirectly affects ULK complex via modulating mTORC1 activation. As a key regulator of cell growth, cell motility, metabolism and protein synthesis, the control of mTORC1 signaling is highly sophisticated. We detected a transient increase in the phosphorylation of $\mathrm{mTOR}$ and p70S6K in MTX-treated SCC-15 cells. It is unclear if the duration of mTORC1 signaling may determine distinct cellular effects in SCC cells. The molecular mechanism by which WWOX regulates $\mathrm{mTORC} 1$ signaling in SCC cells is currently under investigation.

\section{Materials and Methods}

Cell lines and chemicals. SCC-4, -9 and -15 cells derived from human tongue biopsies ${ }^{24}$ were kind gifts from Dr. Dar-Bin Shieh (Department of Oral Medicine, National Cheng Kung University Medical College, Taiwan). Cells were cultured at $37^{\circ} \mathrm{C}$ in $5 \% \mathrm{CO}_{2}$ in a $1: 1$ mixture of Dulbecco's modified Eagle's medium and nutrient mixture F12 (Invitrogen, Carlsbad, CA, USA) supplemented with $400 \mathrm{ng} / \mathrm{ml}$ hydrocortisone (Sigma, St. Louis, MO), $2 \mathrm{~mm}$ L-glutamine, $0.5 \mathrm{~mm}$ sodium pyruvate and $10 \%$ fetal bovine serum. Pepstatin $A$ and (2S,3S)-transepoxysuccinyl--Lleucylamido-3-methylbutane ethyl ester (E64d) were from Tocris (Bristol, UK) and Sigma, respectively.

Cell death assay. SCC cells were cultured in 96-well plates with or without the treatment of MTX (Calbiochem, Darmstadt, Grmany). After the indicated time intervals, cells were stained with $0.5 \%$ crystal violet in $20 \%$ methanol at room temperature for $15 \mathrm{~min}$. Unbound dye and detached cells were removed by washing. Bound crystal violet was dissolved with $33 \%$ acetic acid (in $100 \mu \mathrm{l}$ ) and quantified by optical density determination at $590 \mathrm{~nm}$ using an ELISA reader. The percentages of viable cells were calculated as follows: $\left(\mathrm{OD}_{590}\right.$ of MTX-treated cells $/ \mathrm{OD}_{590}$ of untreated cells) $\times 100 \%$. For detecting the exposure of phosphatidylserine on cell surface during apoptosis, the binding of FITCconjugated annexin V (Calbiochem) to SCC cells was determined by fluorescence microscopy (Olympus, Tokyo, Japan) according to the manufacturer's instruction. 
Co-immunoprecipitation and western blot analysis. Whole-cell lysates were prepared using a lysis buffer containing $0.1 \%$ SDS, $1 \%$ nonidet $\mathrm{P}-40$, $0.5 \%$ Tween $20,10 \mathrm{mM} \mathrm{Na}_{3} \mathrm{VO}_{4}, 10 \mathrm{~mm} \mathrm{Na}_{4} \mathrm{P}_{2} \mathrm{O}_{7}, 10 \mathrm{~mm} \mathrm{NaF}$ and $1: 20$ dilution of protease inhibitor cocktail (Sigma) in PBS, and the lysates were collected after centrifugation at $13000 \times g$ for $10 \mathrm{~min}$ at $4^{\circ} \mathrm{C}$. Total protein concentrations were determined using Bio-Rad Protein Assay Dye Reagent (Hercules, CA, USA). For co-immunoprecipitation and western blot experiments, we have generated rabbit polyclonal antibody against the N-terminal WW domains of human WWOX protein using an EZ antibody production and purification kit (Pierce, Rockford, IL, USA). ${ }^{18}$ This antibody has been demonstrated to recognize a $46-k D a$ full-length WWOX protein. ${ }^{18}$ Co-immunoprecipitation was performed as described previously. ${ }^{18}$ The precipitated proteins or equal amounts of whole-cell lysates were separated by SDS-PAGE and transferred to PVDF membranes. Additional antibodies used in Western blot analysis were against the following proteins: caspase-3, caspase-9, mTOR, phospho-mTOR, p70S6K, phospho-p70S6K, Beclin-1 (Cell Signaling, Danvers, MA, USA), LC3 (Medical \& Biological Laboratories, Woburn, MA, USA), Atg5 (Abnova, Taipei, Taiwan), GFP (Abcam, Cambridge, UK) and $\beta$-actin (Sigma). Horseradish peroxidase-conjugated goat anti-rabbit IgG or horse antimouse IgG (Cell Signaling) was used as secondary antibody. Antibody probing and enhanced chemiluminescence (ECL, Amersham, Buckinghamshire, UK) detection were performed as previously described. ${ }^{25}$ Quantitative densitometry of immunoblots was performed using the ImageJ program (National Institutes of Health, Bethesda).

Reverse transcription PCR. RNA was extracted using TRIZOL reagent (Invitrogen) according to the manufacturer's instruction. Reverse transcription PCR was performed as described earlier. ${ }^{19}$ The PCR primer pairs and amplification protocols used were as follows: (1) human WWOX, 5'-AAAACGACTATTGGGC GATG-3' (forward) and 5'-GTGTTGGAGGGACATTTGGA-3' (reverse), 35 cycles at $95^{\circ} \mathrm{C}$ for $1 \mathrm{~min}, 65^{\circ} \mathrm{C}$ for $1 \mathrm{~min}$ and $72^{\circ} \mathrm{C}$ for $1 \mathrm{~min}$, followed by $72^{\circ} \mathrm{C}$ for $10 \mathrm{~min}$ for the final extension and (2) human $\beta$-actin, $5^{\prime}$-AGCGG GAAATCGTGCGTG-3' (forward) and 5'-CAGGGTACATGGTGGTG-3' (reverse), 26 cycles at $95^{\circ} \mathrm{C}$ for $1 \mathrm{~min}, 60^{\circ} \mathrm{C}$ for $1 \mathrm{~min}$ and $72{ }^{\circ} \mathrm{C}$ for $1 \mathrm{~min}$, followed by $72{ }^{\circ} \mathrm{C}$ for $10 \mathrm{~min}$ for the final extension. The PCR fragments were subjected to electrophoresis on $1.2 \%$ agarose gels run in $1 \times \mathrm{TAE}$, visualized by ethidium bromide staining and analyzed using the ImageQuant 300 imaging system (GE Healthcare Life Sciences, Buckinghamshire, UK). For quantitative real-time PCR analysis, $5 \mu \mathrm{g}$ of total RNA extracted from SCC- 9 and SCC- 15 cells were reverse transcribed at $37^{\circ} \mathrm{C}$ for $2 \mathrm{~h}$ using $0.5 \mu \mathrm{g}$ of oligo(dT) 15 primer, $0.5 \mathrm{mM}$ dNTPs, $5 \times$ first strand buffer and $1 \mathrm{U}$ of Moloney murine leukemia virus reverse transcriptase (Promega, Madison, WI, USA) in a final reaction volume of $50 \mu \mathrm{l}$. Real-time PCR was performed using a LightCycler 1.5 instrument (Roche Applied Science, Mannheim, Germany) with the SYBR Advantage qPCR Premix (Clontech, Mountain View, CA, USA) according to the manufacturer's instructions. The primer sequences used were as described above. The amplification and melting curves of the end products were obtained to confirm the PCR specificity. Agarose gel electrophoresis was performed to verify the size of PCR products. All real-time PCR tests were performed in triplicate. Results were analyzed using the comparative $C t$ method, where $\Delta C t=C t$ (WWOX) $-C t$ ( $\beta$-actin). The relative amount of WWOX mRNA was calculated by normalizing the $\Delta C t$ value obtained from each sample with that from the untreated SCC-15 cells.

Immunohistochemistry and TUNEL assay. Tumor biopsies from SCC patients treated with continuous intra-arterial infusion of MTX were obtained from the Kaohsiung Medical University Chung-Ho Memorial Hospital, Taiwan, under a protocol approved by the Institutional Review Board. ${ }^{21,22}$ Immunohistochemical staining of $5-\mu \mathrm{m}$ thick tissue sections was performed as described. ${ }^{19}$ Antibodies against $\mathrm{N}$-terminal region between the first and second WW domains of WWOX ${ }^{18,19,38}$ and cleaved caspase-3 (Cell Signaling) were used for staining. For TUNEL assay, formalin-fixed tissue sections were deparaffinized and treated with proteinase $\mathrm{K}(20 \mu \mathrm{g} / \mathrm{ml})$ at room temperature for $15 \mathrm{~min}$. The tissue sections were treated with $3 \%$ hydrogen peroxide in PBS for $5 \mathrm{~min}$ to quench endogenous peroxidase activity. After wash, incorporation of digoxigenin-conjugated nucleotides into the DNA strand breaks in apoptotic cells was performed by incubating the sections with terminal deoxynucleotidyl transferase in the reaction buffer at $37^{\circ} \mathrm{C}$ for $1 \mathrm{~h}$ (Chemicon, Temecula, CA, USA). The sections were then stained with a horseradish peroxidase-conjugated anti-digoxigenin antibody, and TUNEL-positive cells were visualized using the chromogen diaminobenzidine as substrate under an Olympus light microscope.
Expression constructs, siRNA, lentiviral shRNA and gene transfection. The expression constructs for GFP-tagged human WWOX and dnWWOX in pEGFP-C1 (Clontech) were made as previously described. ${ }^{18}$ The dnWWOX mutant has been demonstrated to block p53 activation and apoptosis. ${ }^{18}$ The plasmid encoding GFP-tagged LC3 was a kind gift from Dr. Noboru Mizushima (Department of Physiology and Cell Biology, Tokyo Medical and Dental University, Japan). A plasmid construct for generating the siRNA targeting human WWOX (WWOXsi) was made using a mammalian expression vector, pSuppressorNeo (Imgenex, San Diego, CA, USA). Synthetic primers for making this WWOXsi construct were as follows: forward, $5^{\prime}$-TCGAGCCAAGTC CATGCACAGGGGAGTACTGCCCTGTTGCATGGACTTGGTTTT- $3^{\prime}$ and reverse, 05'-CTAGAAAAACCAAGTCCATGCACAGGGCAGTACTCCCCTGTTGCATGGA CTTGGC- $3^{\prime}$. The vector with a scrambled sequence from Imgenex was used as a negative control. This WWOXsi-expressing construct has been demonstrated to suppress WWOX protein expression by $>50 \%$ compared with the control scrambled siRNA. ${ }^{18,19}$ Where indicated, a lentiviral shRNA expression system from System Biosciences (Mountain View, CA, USA) was used to suppress WWOX expression according to the manufacturer's instruction. The template oligonucleotide sequences used were 5'-GATCCCCAAGTCCATGCAACAGGGCTTCCTGT CAGACCCTGTTGCATGGACTTGGTTTTTG- $3^{\prime}$ and $5^{\prime}$-AATTCAAAAACCAAGTC CATGCAACAGGGTCTGACAGGAAGCCCTGTTGCATGGACTTGGG-3'. For transfection of plasmid DNA, cells were electroporated with the above constructs or a control vector (200 V and $50 \mathrm{mSec}$, square wave; Bio-Rad Gene Pulser System) and cultured in 96-well plates or 10-cm dishes. Twenty-four hours later, these cells were treated with or without $10 \mu \mathrm{g} / \mathrm{ml}$ MTX and cultured for 24 or $48 \mathrm{~h}$ for determining protein expression and phosphorylation, and 72 or $96 \mathrm{~h}$ for cell death assay.

Confocal microscopy. Cells on glass coverslips were washed with cold PBS, fixed with $3.7 \%$ formaldehyde/PBS at $4{ }^{\circ} \mathrm{C}$ for $20 \mathrm{~min}$ and washed twice with PBS. The samples were mounted on a slide and visualized under a laser-scanning confocal microscope (Olympus FV1000).

Transmission electron microscopy. SCC cells were fixed at $4{ }^{\circ} \mathrm{C}$ overnight using a freshly prepared $4 \%$ glutaraldehyde/ $0.1 \mathrm{M}$ cacodylate buffer, dehydrated in a graded series of ethanol and finally embedded in resin (EMS; SPUR's Kit). Ultrathin sections $(70-80 \mathrm{~nm})$ were prepared with an ultramicrotome (Reichert-Jung, Vienna, Austria). The sections were stained with saturated aqueous uranyl acetate and lead citrate at room temperature. Specimens were observed under a transmission electron microscopy (JEOL JEM-1200EX, Tokyo, Japan) at $100 \mathrm{kV}$.

Statistical analysis. Data were presented as the means \pm S.D. Statistical significance was determined using Student's $t$-test. The differences were considered significant when the $P$-values were $<0.05$.

\section{Conflict of Interest}

The authors declare no conflict of interest.

Acknowledgements. We thank Kai-Fei Chang, Yu-Han Su and Hui-Ching Cheng for helping us with data collection and statistical analysis. This study was supported by the following: (1) the National Science Council, Taiwan, grants NSC95-2320-B-006-072-MY2, 97-2314-B-006-064, 98-2320-B-006-007-MY3 and 101-2320-B-006-032-MY3 to L-JH; (2) the National Health Research Institute, Taiwan, grant NHRI-EX97-9705BI to N-SC; (3) the Department of Defense, USA, grant W81XWH-08-1-0682 to N-SC; (4) Chi-Mei Hospital and National Cheng Kung University Collaborative Research grants CMNCKU9902, CMNCKU10008 and CMNCKU10204 to F-JL and L-JH; and (5) the Department of Health, Taiwan, grant DOH99-TD-C-111-003 to L-JH.

1. Colevas $A D$. Chemotherapy options for patients with metastatic or recurrent squamous cell carcinoma of the head and neck. J Clin Oncol 2006; 24: 2644-2652.

2. Genestier L, Paillot R, Quemeneur L, Izeradjene K, Revillard JP. Mechanisms of action of methotrexate. Immunopharmacology 2000; 47: 247-257.

3. Mizushima N. Autophagy: process and function. Genes Dev 2007; 21: 2861-2873.

4. Baehrecke EH. Autophagy: dual roles in life and death? Nat Rev Mol Cell Biol 2005; 6 : 505-510. 
5. Lum JJ, Bauer DE, Kong M, Harris MH, Li C, Lindsten T et al. Growth factor regulation of autophagy and cell survival in the absence of apoptosis. Cell 2005; 120: 237-248.

6. Levine B. Autophagy and cancer. Nature 2007; 446: 745-747.

7. Li J, Hou N, Faried A, Tsutsumi S, Takeuchi T, Kuwano H. Inhibition of autophagy by 3-MA enhances the effect of 5-FU-induced apoptosis in colon cancer cells. Ann Surg Oncol 2009; 16: 761-771.

8. Bijnsdorp IV, Peters GJ, Temmink OH, Fukushima M, Kruyt FA. Differential activation of cell death and autophagy results in an increased cytotoxic potential for trifluorothymidine compared to 5-fluorouracil in colon cancer cells. Int J Cancer 2010; 126: 2457-2468.

9. Carew JS, Nawrocki ST, Cleveland JL. Modulating autophagy for therapeutic benefit. Autophagy 2007; 3: 464-467.

10. Song J, Qu Z, Guo X, Zhao Q, Zhao X, Gao L et al. Hypoxia-induced autophagy contributes to the chemoresistance of hepatocellular carcinoma cells. Autophagy 2009; $\mathbf{5}$ : $1131-1144$.

11. Kang R, Tang D, Schapiro NE, Livesey KM, Farkas A, Loughran $P$ et al. The receptor for advanced glycation end products (RAGE) sustains autophagy and limits apoptosis, promoting pancreatic tumor cell survival. Cell Death Differ 2010; 17: 666-676.

12. Chang NS, Hsu LJ, Lin YS, Lai FJ, Sheu HM. WW domain-containing oxidoreductase: a candidate tumor suppressor. Trends Mol Med 2007; 13: 12-22.

13. Jenner MW, Leone PE, Walker BA, Ross FM, Johnson DC, Gonzalez D et al. Gene mapping and expression analysis of $16 q$ loss of heterozygosity identifies WWOX and CYLD as being important in determining clinical outcome in multiple myeloma. Blood 2007; 110: $3291-3300$

14. Ramos D, Abba M, López-Guerrero JA, Rubio J, Solsona E, Almenar S et al. Low levels of WWOX protein immunoexpression correlate with tumour grade and a less favourable outcome in patients with urinary bladder tumours. Histopathology 2008; 52 : 831-839.

15. Fabbri M, lliopoulos D, Trapasso F, Aqeilan RI, Cimmino A, Zanesi N et al. WWOX gene restoration prevents lung cancer growth in vitro and in vivo. Proc Natl Acad Sci USA 2005; 102: $15611-15616$

16. Iliopoulos D, Fabbri M, Druck T, Qin HR, Han SY, Huebner K. Inhibition of breast cancer cell growth in vitro and in vivo: effect of restoration of Wwox expression. Clin Cancer Res 2007; 13: 268-274.

17. Nakayama S, Semba S, Maeda N, Aqeilan RI, Huebner K, Yokozaki H. Role of the WWOX gene, encompassing fragile region FRA16D, in suppression of pancreatic carcinoma cells. Cancer Sci 2008; 99: 1370-1376.

18. Chang NS, Doherty J, Ensign A, Schultz L, Hsu LJ, Hong Q. WOX1 is essential for tumor necrosis factor-, UV light-, staurosporine-, and p53-mediated cell death, and its tyrosine 33-phosphorylated form binds and stabilizes serine 46-phosphorylated p53. J Biol Chem 2005; 280: 43100-43108

19. Lai FJ, Cheng CL, Chen ST, Wu CH, Hsu LJ, Lee JY et al. WOX1 is essential for UVB irradiation-induced apoptosis and down-regulated via translational blockade in UVBinduced cutaneous squamous cell carcinoma in vivo. Clin Cancer Res 2005; 11: 5769-5777.

20. Aqeilan RI, Trapasso F, Hussain S, Costinean S, Marshall D, Pekarsky Y et al. Targeted deletion of Wwox reveals a tumor suppressor function. Proc Natl Acad Sci USA 2007; 104: 3949-3954.

21. Sheen MC, Sheu HM, Lai FJ, Lin SD, Wu CF, Wang YW et al. A huge verrucous carcinoma of the lower lip treated with intra-arterial infusion of methotrexate. Br J Dermatol 2004; 151 : 727-729.

22. Wu CF, Chen $\mathrm{CM}$, Chen $\mathrm{CH}$, Shieh TY, Sheen MC. Continuous intraarterial infusion chemotherapy for early lip cancer. Oral Oncol 2007; 43: 825-830.
23. Wu CF, Chen CM, Shen YS, Huang IY, Chen $\mathrm{CH}$, Chen $\mathrm{CY}$ et al. Effective eradication of oral verrucous carcinoma with continuous intraarterial infusion chemotherapy. Head Neck 2008; 30: 611-617.

24. Rheinwald JG, Beckett MA. Tumorigenic keratinocyte lines requiring anchorage and fibroblast support cultures from human squamous cell carcinomas. Cancer Res 1981; 41: 1657-1663.

25. Hsu LJ, Schultz L, Hong Q, Van Moer K, Heath J, Li MY et al. Transforming growth factor $\beta 1$ signaling via interaction with cell surface Hyal-2 and recruitment of WWOX/WOX1. J Biol Chem 2009; 284: 16049-16059.

26. Degenhardt K, Mathew R, Beaudoin B, Bray K, Anderson D, Chen G et al. Autophagy promotes tumor cell survival and restricts necrosis, inflammation, and tumorigenesis. Cancer Cell 2006; 10: 51-64.

27. Mizushima N, Yoshimori T, Levine B. Methods in mammalian autophagy research. Cell 2010; 140: 313-326.

28. Kanazawa T, Taneike I, Akaishi R, Yoshizawa F, Furuya N, Fujimura S et al. Amino acids and insulin control autophagic proteolysis through different signaling pathways in relation to mTOR in isolated rat hepatocytes. J Biol Chem 2004; 279: 8452-8459.

29. Mizushima N, Sugita H, Yoshimori T, Ohsumi Y. A new protein conjugation system in human: The counterpart of the yeast Apg12p conjugation system essential for autophagy. J Biol Chem 1998; 273: 33889-33892.

30. Hanada T, Noda NN, Satomi Y, Ichimura Y, Fujioka Y, Takao T et al. The Atg12-Atg5 conjugate has a novel E3-like activity for protein lipidation in autophagy. J Biol Chem 2007; 282: 37298-37302.

31. Furuya N, Yu J, Byfield M, Pattingre S, Levine B. The evolutionarily conserved domain of Beclin 1 is required for Vps34 binding, autophagy and tumor suppressor function. Autophagy 2005; 1: 46-52.

32. Salah Z, Bar-mag T, Kohn Y, Pichiorri F, Palumbo T, Melino G et al. Tumor suppressor WWOX binds to $\triangle \mathrm{Np} 63 \alpha$ and sensitizes cancer cells to chemotherapy. Cell Death Dis 2013; 4: e480.

33. Aqeilan RI, Donati V, Gaudio E, Nicoloso MS, Sundvall M, Korhonen A et al. Association of Wwox with ErbB4 in breast cancer. Cancer Res 2007; 67: 9330-9336.

34. Kuroki T, Yendamuri S, Trapasso F, Matsuyama A, Aqeilan Rl, Alder $\mathrm{H}$ et al. The tumor suppressor gene WWOX at FRA16D is involved in pancreatic carcinogenesis. Clin Cancer Res 2004; 10: 2459-2465.

35. Iliopoulos D, Guler G, Han SY, Johnston D, Druck T, McCorkell KA et al. Fragile genes as biomarkers: epigenetic control of WWOX and FHIT in lung, breast and bladder cancer. Oncogene 2005; 24: 1625-1633.

36. Cantor JP, Iliopoulos D, Rao AS, Druck T, Semba S, Han SY et al. Epigenetic modulation of endogenous tumor suppressor expression in lung cancer xenografts suppresses tumorigenicity. Int J Cancer 2007; 120: 24-31.

37. Guler G, lliopoulos D, Guler N, Himmetoglu C, Hayran M, Huebner K. Wwox and Ap2 $\gamma$ expression levels predict tamoxifen response. Clin Cancer Res 2007; 13: 6115-6121.

38. Mizushima N. The role of the Atg1/ULK1 complex in autophagy regulation. Curr Opin Cell Biol 2010; 22: 132-139.

(1) () () Cell Death and Disease is an open-access journal published by Nature Publishing Group. This work is licensed under a Creative Commons Attribution-NonCommercialNoDerivs 3.0 Unported License. To view a copy of this license, visit http://creativecommons.org/licenses/by-nc-nd/3.0/

Supplementary Information accompanies this paper on Cell Death and Disease website (http://www.nature.com/cddis) 